\title{
Manejo de los escarabajos perforadores Monochamus galloprovincialis (Olivier) y M. sutor (Linnaeus) mediante compuestos semioquímicos
}

\author{
Álvarez Baz, G. * \\ Instituto de Investigación en Gestión Forestal Sostenible, Universidad de Valladolid. \\ Campus de La Yutera. Avda. de Madrid, 44. 34004, Palencia. \\ *e-mail: gonzalbaz@gmail.com
}

\section{Introducción}

Monochamus galloprovincialis y M. sutor son plagas secundarias de pinos presentes en Europa y Norte de África. El primero de ellos ha sido identificado como el vector en Europa del nematodo de la madera del pino Bursaphelenchus xylophilus (Sousa et al., 2001), siendo el segundo probablemente capaz de transmitirlo también (Evans et al., 1996). Este nematodo es el causante de la enfermedad del marchitamiento de los pinos (Wingfield, 1982), que ha causado graves pérdidas en los países donde ha sido introducido (Shin, 2008; Zhao, 2008). En Europa se ha extendido por gran parte de Portugal (Daub, 2008; Rodrigues, 2008) y en España se han detectado cuatro focos desde su introducción en 2008 (Espárrago, 2012). El manejo de la enfermedad a través de sus insectos vectores se considera una de las estrategias más prometedoras y el uso de trampas cebadas con atrayentes específicos ha mostrado ser una alternativa eficaz y preferible al uso de insecticidas. La presente tesis aporta avances importantes en el desarrollo de trampas y cebos atrayentes eficaces para estas especies así como en la aplicación de estas herramientas al control biológico con hongos entomopatógenos, mediante técnicas de autodiseminación. 


\section{Desarrollo de trampas para la captura de M. galloprovincialis vivos}

\subsection{Antecedentes}

Un sistema de trampeo eficaz puede ser útil no sólo para el monitoreo del insecto vector sino también para el control directo de su población. El trampeo, además, puede proporcionar información clave sobre la carga de nematodos transportados por los insectos siempre que éstos permanezcan vivos en la trampa, permitiendo la detección temprana de focos de infección (Schroeder, 2012). Los programas de trampeo de Monochamus han utilizado tanto trampas multiembudo (Lindgren, 1983) como de intercepción (Graham et al., 2012a). Aunque trabajos previos habían comparado la eficacia de estos dos diseños fundamentales, no quedaba claro si alguno de ellos era más apropiado para capturar insectos tan ágiles como Monochamus. Lo que sí se daba por probado es que los abundantes bordes y superficies de estas trampas facilitaban que los insectos atraídos escaparan antes de caer en su interior (Graham et al., 2012b). En nuestro estudio se desarrollaron distintos diseños de trampas (Figura 1) y se compararon con los modelos estándar comerciales para determinar cuáles maximizaban el número de insectos capturados y la proporción de ellos que permanecía viva. En total se evaluaron 12 modelos de trampas en cinco experimentos de campo realizados en Francia, España y Portugal (Tabla1).

(a)

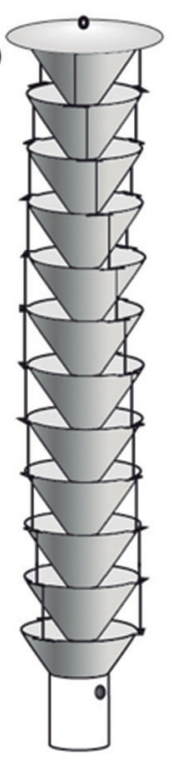

(b)

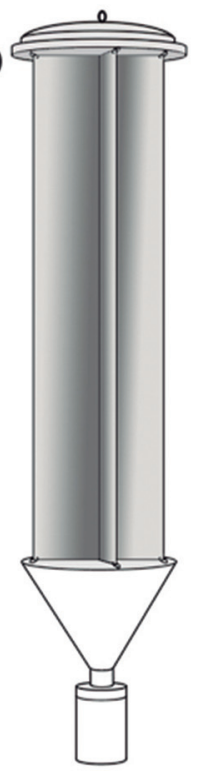

(c)

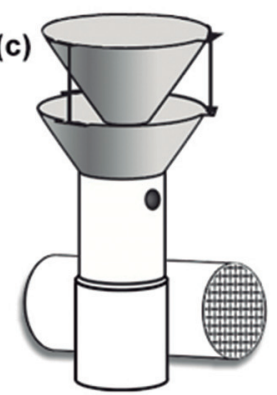

(d)

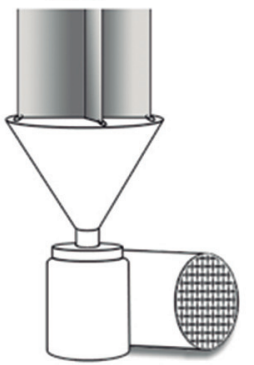

(e)

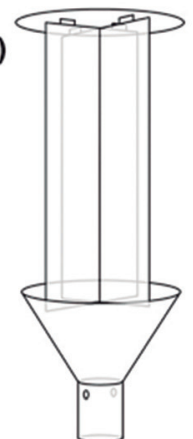

(f)

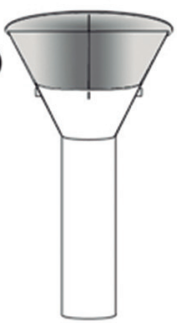

(g)

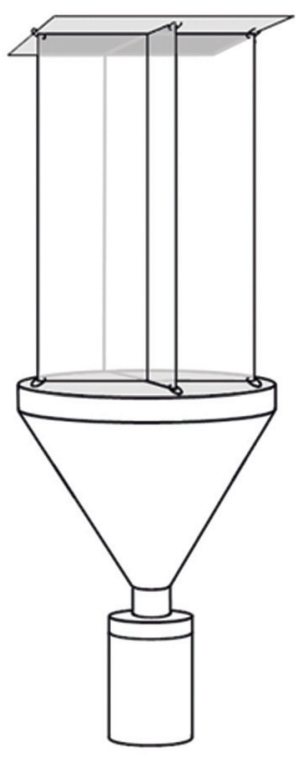

Figura 1. Diseños de trampas probados en los experimentos: (a) Multiembudo, (b) intercepción, (c) colector extendido en trampa multiembudo con fondo de malla, (d) colector extendido en trampa de intercepción con fondo de malla, (e) Polytrap ${ }^{\circledR}$, (f) MasTrap L ${ }^{\circledR}$, (g) trampa de intercepción transparente. 
Tabla 1. Descripción, proveedor, país y año del ensayo en el que se evaluó cada tipo de trampa.

\begin{tabular}{|c|c|c|}
\hline Descripción & Proveedor & Año / País \\
\hline $\begin{array}{l}\text { Multiembudo con colector } \\
\text { recubierto de Fluón y fondo de malla }\end{array}$ & $\begin{array}{l}\text { Modificada a mano } \\
\text { por Econex (Murcia, España) }\end{array}$ & 2010 / España \\
\hline $\begin{array}{l}\text { Multiembudo totalmente recubierta } \\
\text { de Fluón }\end{array}$ & $\begin{array}{l}\text { Modificada a mano } \\
\text { por Econex (Murcia, España) }\end{array}$ & 2010 / España \\
\hline $\begin{array}{l}\text { Multiembudo con colector } \\
\text { recubierto de Fluón }\end{array}$ & $\begin{array}{l}\text { Modificada a mano } \\
\text { por Econex (Murcia, España) }\end{array}$ & 2010 / España \\
\hline Multiembudo & $\begin{array}{l}\text { Proporcionada por Econex } \\
\text { (Murcia, España) }\end{array}$ & 2010 / España \\
\hline Intercepción con DDVP* & $\begin{array}{l}\text { Proporcionada por Econex } \\
\text { (Murcia, España) }\end{array}$ & 2010 / España \\
\hline Multiembudo con DDVP* & $\begin{array}{l}\text { Proporcionada por Econex } \\
\text { (Murcia, España) } \\
\text { y Contech (Loc,Canada) }\end{array}$ & $\begin{array}{l}2010 \text { / España } \\
2011 \text { / Francia, } \\
\text { Portugal y España }\end{array}$ \\
\hline $\begin{array}{l}\text { Intercepción totalmente recubierta } \\
\text { de Teflón y colector extendido }\end{array}$ & $\begin{array}{l}\text { Proporcionada por Econex } \\
\text { (Murcia, España) }\end{array}$ & $\begin{array}{l}2011 \text { / Francia, Portugal } \\
\text { y España; } 2012 \text { / España }\end{array}$ \\
\hline $\begin{array}{l}\text { Multiembudo totalmente recubierta } \\
\text { de Teflón y colector extendido }\end{array}$ & $\begin{array}{l}\text { Proporcionada por Econex } \\
\text { (Murcia, España) }\end{array}$ & 2011, 2012 / España \\
\hline Polytrap $^{\circledR}$ con DDVP* & $\begin{array}{l}\text { Proporcionada por Purpan } \\
\text { Engineering School } \\
\text { (Toulouse, Francia) }\end{array}$ & 2011 / Francia, España \\
\hline MasTrap $\mathrm{L}^{\circledR}$ con DDVP* & $\begin{array}{l}\text { Proporcionada por Biotop } \\
\text { (Livron sur Drôme, Francia) }\end{array}$ & 2011 / Francia \\
\hline $\begin{array}{l}\text { Multiembudo totalmente recubierta } \\
\text { de Tefón }\end{array}$ & $\begin{array}{l}\text { Proporcionada por Econex } \\
\text { (Murcia, España) }\end{array}$ & 2011 / Portugal \\
\hline Intercepción transparente & Hecha a mano & 2011 / Portugal \\
\hline
\end{tabular}

* DDVP: 2,2-diclorovinil-dimetil fosfato (insecticida).

\subsection{Resultados}

El recubrimiento de Teflón aplicado a toda la trampa y el uso de botes colectores extendidos y con ventilación (fondo de malla) mejoraron significativamente el rendimiento de las trampas permitiendo un aumento del número de capturas de hasta el $275 \%$, en comparación con los diseños estándar. Además, aproximadamente el $30 \%$ de los insectos capturados permanecieron vivos en este tipo de colectores. Estos hallazgos han permitido el desarrollo de nuevos modelos de trampas comerciales (Econex Multifunnel-12 ${ }^{\circledR}$ y Crosstrap ${ }^{\circledR}$; Econex, Murcia, España) disponibles para los gestores forestales. Con los datos recogidos se ajustó un modelo de supervivencia de los insectos en el interior de la trampa, dando como resultado que el tiempo transcurrido entre muestreos consecutivos, así como la humedad relativa y la radiación máxima son las tres variables más influyentes, de 
manera que las trampas pueden proporcionar una muestra útil de insectos vivos si se minimiza su exposición al sol y se muestrean con una periodicidad razonable, no superior a una semana.

\section{Neuronas olfativas receptoras en $M$. galloprovincialis}

\subsection{Antecedentes}

Hasta ahora se sabía que $M$. galloprovincialis respondía a algunos volátiles emitidos por árboles hospedantes y escolítidos de los pinos (Pajares et al., 2004; Ibeas et al., 2007, 2008) y se sospechaba que pudiera ser sensible además a compuestos emitidos por madera quemada, dada la frecuencia con la que coloniza árboles decrépitos tras un incendio. También se había descrito 2-undeciloxi-1-etanol como una feromona agregativa de esta especie liberada por los machos y atractiva a ambos sexos (Pajares et al., 2010). Nunca se había abordado el estudio morfológico y fisiológico de los órganos olfativos a nivel de sensila en esta especia aunque sí se había hecho para las especies norteamericanas M. notatus y M. scutellatus (Dyer and Seabrook, 1975). En nuestro trabajo, se estudiaron las respuestas de 32 y 38 neuronas receptoras olfativas de $M$. galloprovincialis machos y hembras respectivamente a varios de estos volátiles (Tabla 2) usando la técnica de electrofisiología de sensila única.

\subsection{Resultados}

La caracterización morfológica mostró que las antenas de M. galloprovincialis albergan varios tipos de sensilas (Figura 2) y presentan similitudes con las de especies norteamericanas del mismo género (Dyer and Seabrook, 1975). Dos campos sensoriales en ambos extremos de cada segmento antenal están recubiertos de sensilas basicónicas, receptores químicos que contienen una o dos neuronas olfativas. No se encontró dimorfismo sexual respecto a la distribución y abundancia de estas sensilas a lo largo de la antena, sugiriendo que juegan un papel similar en ambos sexos. Esta hipótesis es apoyada por el hecho de que ambos sexos se ven igualmente atraídos por distintos volátiles (Pajares et al., 2004; Ibeas et al., 2007; Pajares et al., 2010).

Las neuronas alojadas en las sensilas basicónicas respondieron con excitaciones o inhibiciones a los diferentes volátiles ensayados. Un análisis de agrupamiento jerárquico encontró seis grupos de neuronas de acuerdo al patrón de sus respuestas (Figura 3): seis neuronas resultaron estar claramente especializadas en la recepción de la feromona agregativa. Las respuestas a cairomonas y volátiles de humo fueron menos específicas pero un grupo de nueve células resultó claramente excitado por éstos últimos, lo que apoya la hipótesis de que podrían utilizar estos olores para localizar sus árboles hospedantes. Un grupo de 8 células respondió muy bien al $\alpha$-pineno, $\beta$-pineno y cis-verbenol y otro grupo de 14 células respon- 
Tabla 2. Compuestos sintéticos empleados en los bioensayos.

\begin{tabular}{|c|c|c|c|}
\hline Compuesto & Solvente & Pureza & Proveedor \\
\hline \multicolumn{4}{|l|}{ Feromona de agregación } \\
\hline 2-undeciloxi-1-etanol & n-hexano & $98 \%$ & NRI \\
\hline \multicolumn{4}{|l|}{ Volatiles de plantas hospedantes } \\
\hline$(+)$-Limoneno & n-hexano & $>93 \%$ & SEDQ \\
\hline$(+)$-Canfeno & n-hexano & $>90 \%$ & SEDQ \\
\hline p-Cimeno & n-hexano & $>97 \%$ & SEDQ \\
\hline$\alpha-$ Pineno & n-hexano & $>97 \%$ & SEDQ \\
\hline$\beta$-Mirceno & n-hexano & $>90 \%$ & SEDQ \\
\hline$\beta$-Pineno & n-hexano & $>97 \%$ & SEDQ \\
\hline 3-Careno & n-hexano & $>90 \%$ & SEDQ \\
\hline \multicolumn{4}{|l|}{ Feromonas de insectos escolítidos } \\
\hline 2-Metil-3-buten-2-ol & n-hexano & $>98 \%$ & SEDQ \\
\hline Cis-verbenol & $\mathrm{CH}_{2} \mathrm{Cl}_{2}$ & $97 \%$ & SEDQ \\
\hline Ipsdienol & n-hexano & $93 \%$ & SEDQ \\
\hline Ipsenol & n-hexano & $93 \%$ & SEDQ \\
\hline \multicolumn{4}{|l|}{ Volátiles de humo } \\
\hline 2-Metoxifenol & $\mathrm{CH}_{2} \mathrm{Cl}_{2}$ & $>98 \%$ & SA \\
\hline 4-Metil-2-metoxifenol & $\mathrm{CH}_{2} \mathrm{Cl}_{2}$ & $>98 \%$ & SA \\
\hline 4-Vinil-2-metoxifenol & $\mathrm{CH}_{2} \mathrm{Cl}_{2}$ & $>98 \%$ & SA \\
\hline Eugenol (2-metoxi-4-alilfenol) & $\mathrm{CH}_{2} \mathrm{Cl}_{2}$ & $>98 \%$ & SA \\
\hline Iso eugenol (2- metoxi -4-propenilfenol) & $\mathrm{CH}_{2} \mathrm{Cl}_{2}$ & $99 \%$ & SA \\
\hline Vanilina (4-hidroxi-3-metoxibenzaldehído) & $\mathrm{CH}_{2} \mathrm{Cl}_{2}$ & $>97 \%$ & SA \\
\hline
\end{tabular}

NRI, Natural Resources Institute, Chatham Maritime, Kent, UK. SEDQ, Sociedad Española de Desarrollos Químicos, Barcelona, España. SA, Sigma-Aldrich, Gillingham, Dorset, UK.

dió a un amplio rango de compuestos. El resto de las células (47\%) no respondieron, lo que sugiere que aún pueden desconocerse muchos compuestos semioquímicos relevantes para esta especie.

\section{Cebos eficaces para la captura de M. galloprovincialis}

\subsection{Antecedentes}

En los últimos años se habían desarrollado atrayentes eficaces para la captura de M. galloprovincialis (Ibeas et al., 2007; Pajares et al., 2010) basados en la feromona agregativa de esta especie (2-undeciloxi-1-etanol), volátiles de los pinos como el $\alpha$-pineno y las cairomonas de escolítidos ipsenol y 2-metil-3-buten-2-ol. El objetivo de nuestro estudio fue optimizar esta combinación de volátiles para mejorar su atracción y especificidad, para lo cual se realizaron diez experimentos de campo (nueve en España y uno en Francia). 
A

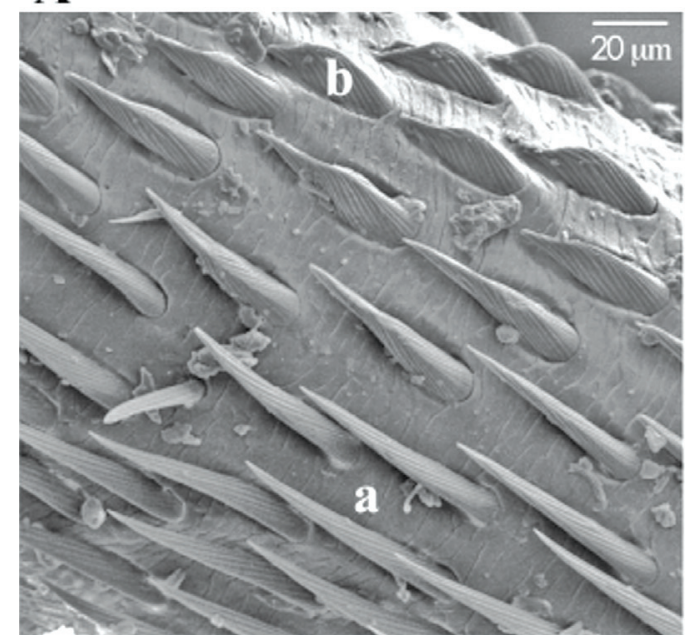

$\mathrm{C}$
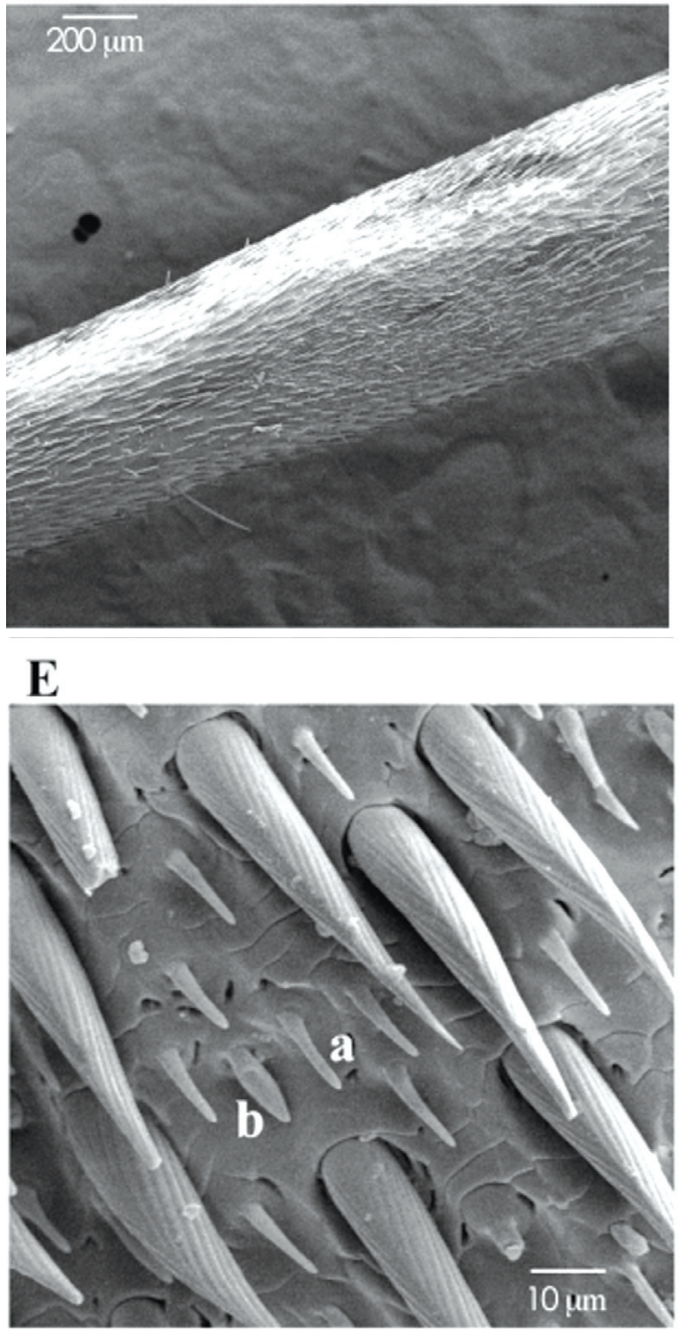

\section{B}

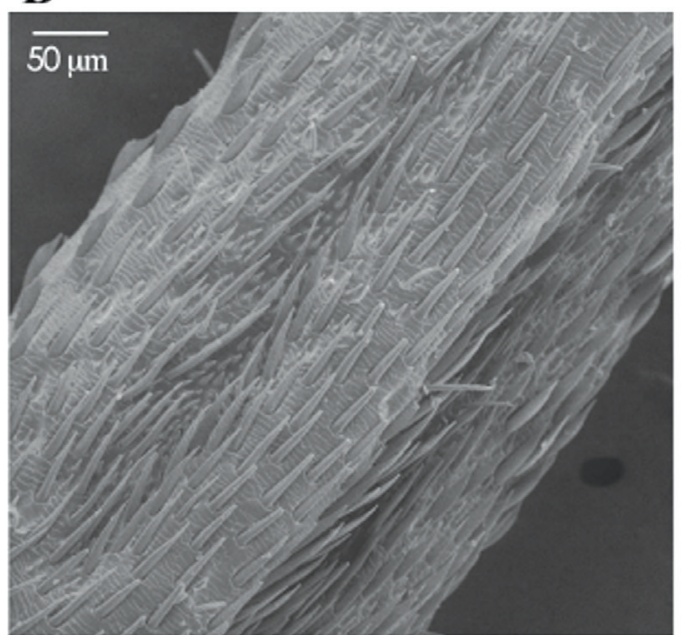

D

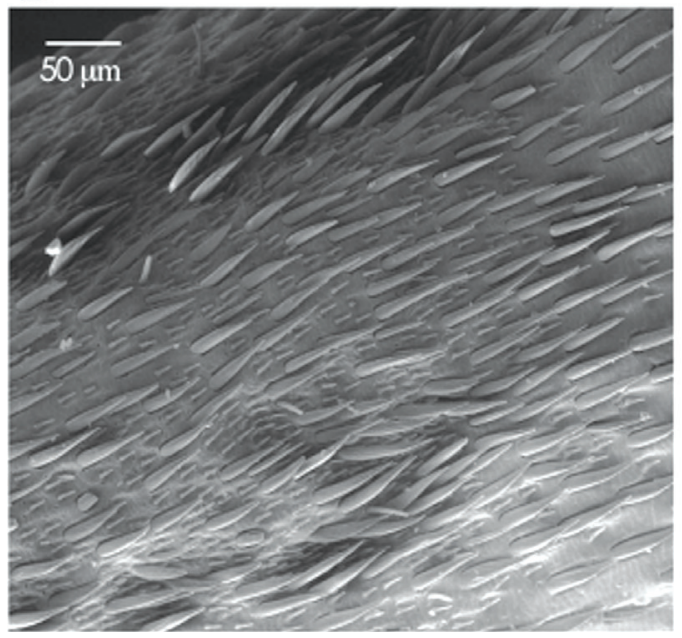

F

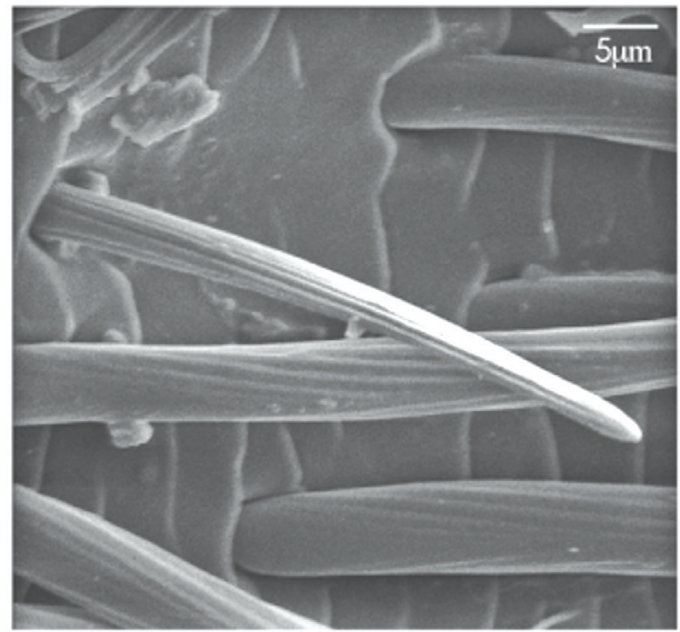



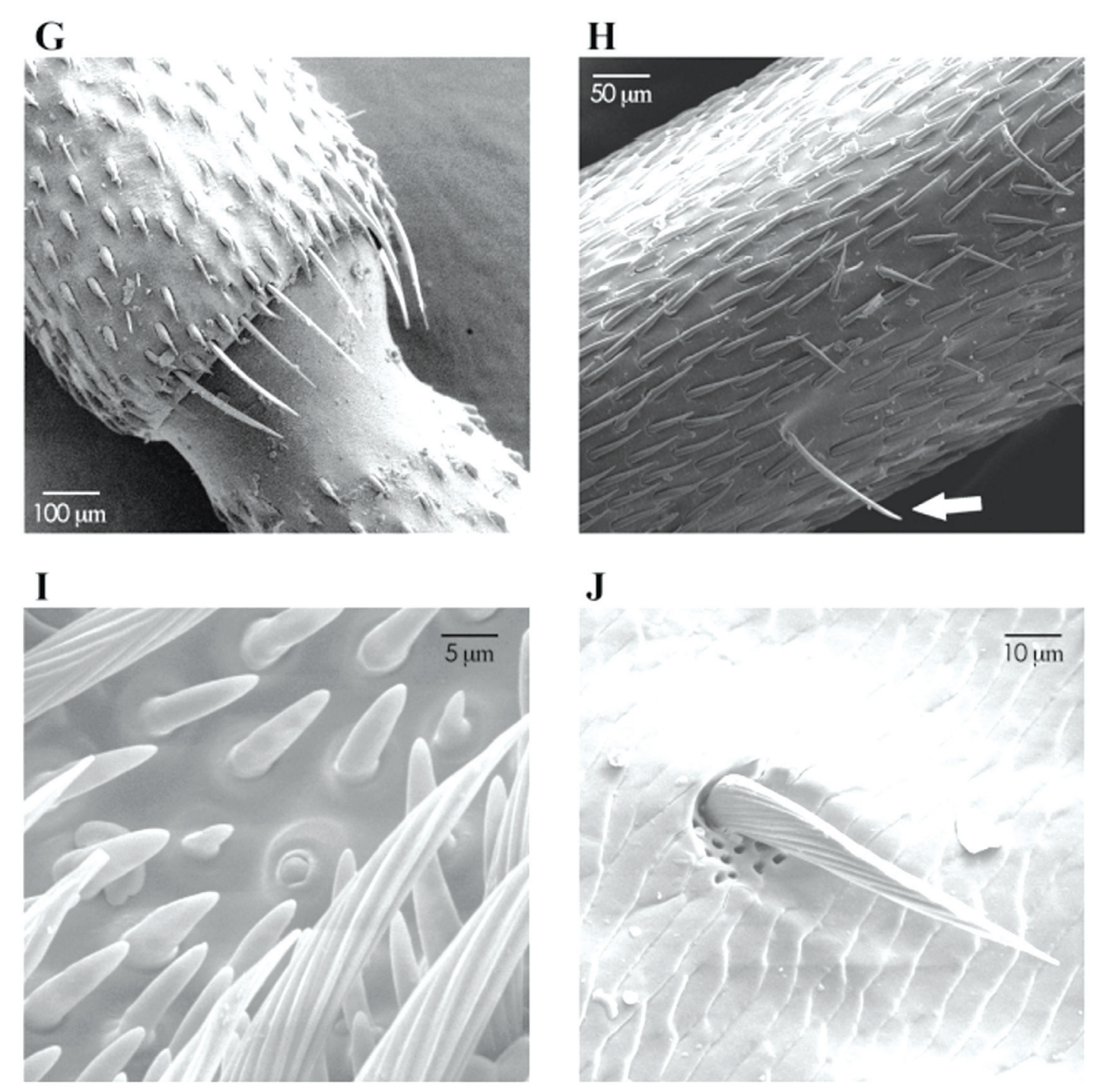

Figura 2. Fotografías de microscopio electrónico de la antena de M. galloprovincialis. (A) Sensila quética gruesa (a) y sensila quética de los machos (b). (B) Campo sensorial acanalado localizado en el extremo proximal de los flagelos (macho). (C) Campo sensorial planiforme localizado en el extremo distal de los flagelos (hembra). (D) Detalle del campo sensorial planiforme recubierto de sensilas basicónicas (macho). (E) Sensila basicónica cilíndrica (a) y aplanada (b). (F) Probablemente sensila tricoidea (hembra). (G) Sensilas quéticas distales. (H) Sensila quética larga (hembra). (I) Órgano cupuliforme (macho). (J) Grupo de poros bajo una sensila quética gruesa (macho).

\subsection{Resultados}

Los experimentos confirmaron que la feromona agregativa de M. galloprovincialis es sinergizada por los volátiles de árboles hospedantes y escolítidos de los pinos, lo que ya se había encontrado en trabajos previos (Pajares et al., 2010; 

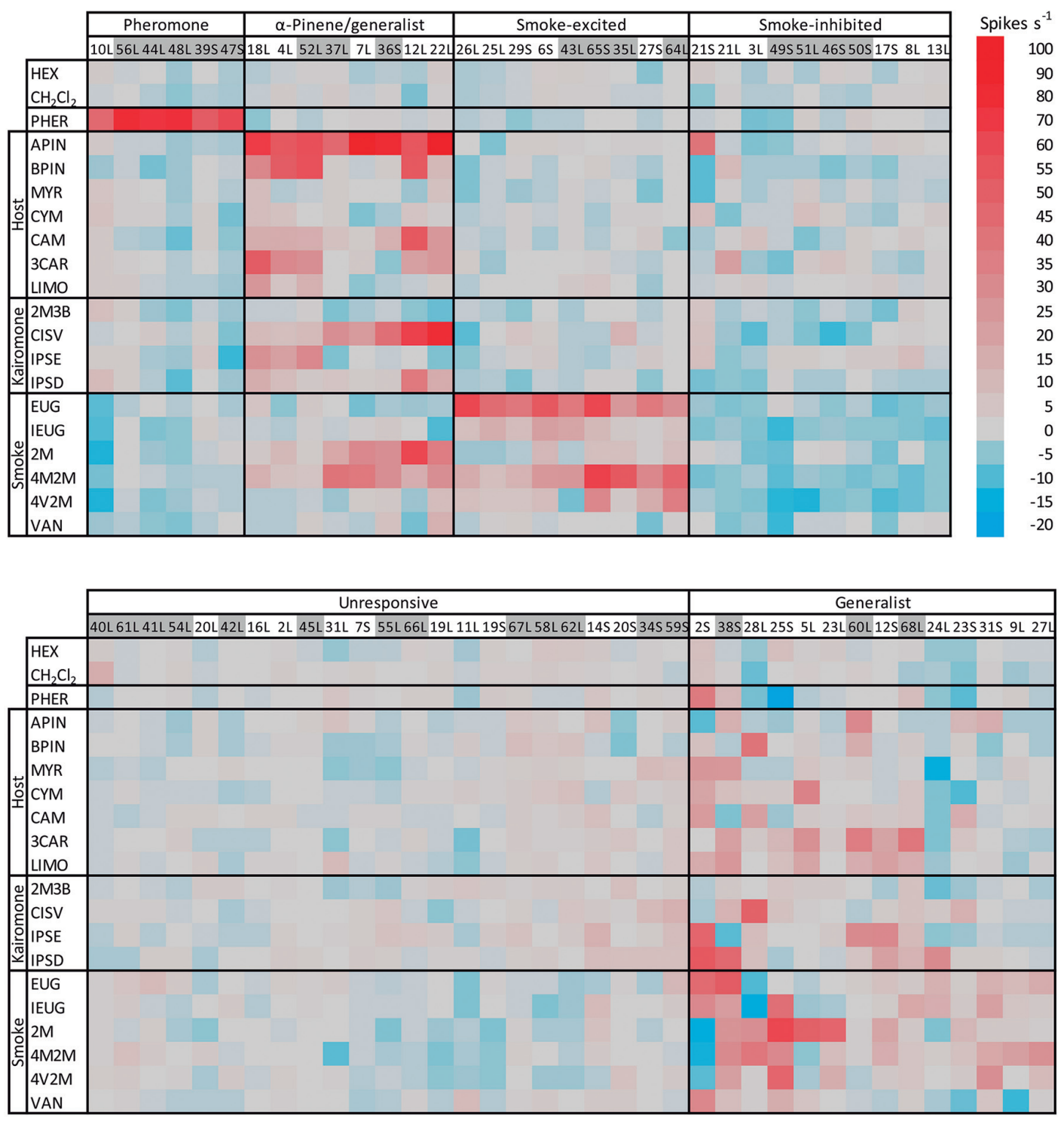

Figura 3. Espectro de respuestas de las neuronas olfativas de Monochamus galloprovincialis a $10 \mu \mathrm{g}$ de los compuestos ensayados. La barra muestra el código de color para las respuestas de inhibición (azul), respuesta pobre o sin respuesta (rango de grises) y excitaciones (en rojo). Las sensilas pueden albergar más de una neurona distinguible por la amplitud de su actividad (pequeña [S] o grande [L]). Identificación de las células en color blanco (hembras) o gris (machos). También se muestran las respuestas a los solventes (n-hexano o $\mathrm{CH}_{2} \mathrm{Cl}_{2}$ ).

Rassati et al., 2012). Se observó una clara relación dosis-respuesta sobre la feromona, con trampas capturando de 2 a 3 veces más individuos cuando se liberó a una tasa de 3 a 5 veces mayor. Este resultado llevó al fabricante (SEDQ, España) a producir un nuevo cebo estándar (Galloprotect 2D), que libera una mayor dosis 
de feromona. Anteriormente se había intentado sustituir sin éxito el $\alpha$-pineno del cebo (Pajares et al., 2010), un volátil de coníferas que atrae depredadores importantes como Temnochila caerulea o Thanasimus formicarius. Nuestros experimentos demostraron que la adición de este terpeno al cebo estándar no mejoraba significativamente las capturas, lo que hace que el uso de este terpeno no sea recomendable. Ninguno de los otros terpenos probados en nuestros experimentos (Figura 4) mejoró significativamente el cebo estándar. Aunque este trabajo ha dado lugar al desarrollo de un nuevo cebo comercial altamente atrayente para los insectos maduros, ninguna de las mezclas ensayadas tuvieron éxito en la atracción de adultos inmaduros. Futuras investigaciones deberán buscar atrayentes para estos insectos.

\section{Cebos eficaces para la captura de M. sutor}

\subsection{Antecedentes}

Se sabía que las cairomonas de escolítidos son atractivas para ambos sexos de M. sutor y se había identificado también 2-undeciloxi-1-etanol como una feromona agregativa de esta especie (Pajares et al., 2013). Este hallazgo abrió la posibilidad de desarrollar un cebo eficaz para este insecto. Los volátiles de humo también podrían jugar un papel relevante en la localización de hospedantes como lo hacen para M. galloprovincialis. Se realizaron electroantenografías sobre $M$. sutor para registrar su respuesta antenal a cairomonas de escolítidos. Posteriormente se llevaron a cabo 11 experimentos de atracción en campo durante tres años en tres países (España, Suecia y Austria), con la finalidad de desarrollar un cebo feromonal-cairomonal operativo y eficaz para el manejo de M. sutor.

\subsection{Resultados}

Los registros de GC-EAG mostraron que ambos sexos de M. sutor respondieron al ipsenol y al ipsdienol, y las hembras además al 2-methyl-3-buten-2-ol y chalcogran. En los ensayos de campo el ipsenol resultó ser la cairomona más atractiva para ambos sexos. Por el contrario, ipsdienol, cis-verbenol y 2-methyl-3-buten-2ol resultaron ser débilmente atractivos y el chalcogran no lo fue en absoluto. Combinados con la feromona agregativa, la mayoría de las cairomonas de escolítidos aumentaron el número de capturas, pero solamente el ipsenol lo hizo de forma significativa. El chalcogran no tuvo ningún efecto. A la vista de estos resultados, el ipsenol no solo se mostró como la cairomona más atractiva de forma individual para M. sutor, sino que también resultó ser la mejor para sinergizar con su feromona. Los resultados con volátiles de humo emitidos por los pinos quemados fueron negativos, lo que sugiere que estas señales probablemente no están implicadas en la búsqueda de árboles hospedantes por esta especie. 


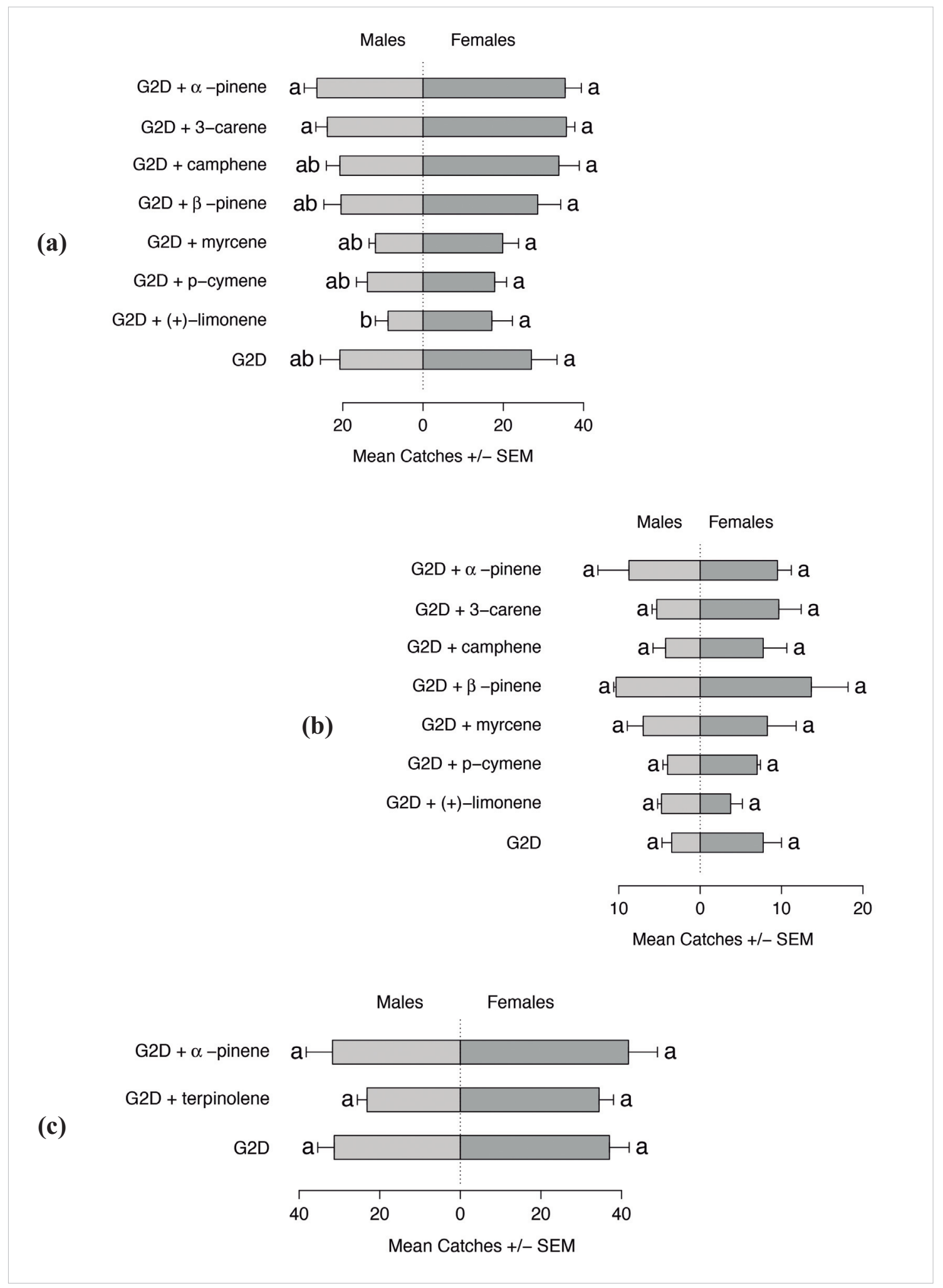

Figura 4. Capturas medias ( \pm SE) de M. galloprovincialis en los experimentos 7 (2011) (a), 8 (2011) (b) and $9 \mathrm{ab}$ (2013) (c). Las barras con la misma letra no son significativamente diferentes (test HSD de Tukey $\operatorname{con} \alpha=0.05)$. 


\section{Hongos entomopatógenos para el control biológico de M. galloprovincialis}

\subsection{Antecedentes}

Algunos estudios habían mostrado que los hongos entomopatógenos pueden ser patógenos naturales comunes de plagas forestales (Draganova, et al., 2013;. Peng et al., 2011;. Sevim et al., 2010). En nuestro trabajo se llevó a cabo un exhaustivo muestreo sobre casi 500 trozas de pinos colonizados de forma natural por M. galloprovincialis con la finalidad de encontrar insectos infectados de los que se pudieran aislar hongos entomopatógenos. Posteriormente se realizaron ensayos de patogenicidad y capacidad de transmisión para evaluar su potencial en el manejo de esta especie.

\subsection{Resultados}

A partir de los ejemplares encontrados se aislaron por primera vez de M. galloprovincialis las tres cepas de hongos entomopatógenos Isaria farinosa (Holmsk.) Fr., Lecanicillium attenuatum (Zare \& W. Games) y Beauveria pseudobassiana (Bals.) Vuill. En los bioensayos de patogenicidad la cepa B. pseudobassiana EABps 11/01$\mathrm{Mg}$ demostró ser el aislamiento más agresivo contra $M$. galloprovincialis, causando una mortalidad del $100 \%$ de los ejemplares inoculados. La efectividad de este hongo en la supresión de la enfermedad del marchitamiento de los pinos depende de su capacidad para matar a los insectos antes del pico de transmisión a los árboles sanos (Shimazu, 2004), de 2 a 6 semanas después de la emergencia (Naves et al., 2007). Por lo tanto se estudió la capacidad de transmisión horizontal (de unos adultos a otros) y vertical (de una generación a la siguiente), tanto con una suspensión de conidios en agua $\left(1 \times 10^{8}\right.$ conidios $\left./ \mathrm{ml}\right)$ como con una preparación seca en polvo de talco $\left(4.25 \times 10^{9}\right.$ conidios $\left./ \mathrm{g}\right)$. No se encontraron evidencias de transmisión cuando se utilizó la suspensión acuosa. Sin embargo, con la preparación seca murieron el $100 \%$ de los insectos infectados por transmisión horizontal y su tiempo medio de vida se redujo significativamente (de 21.10 y 25.00 días en los controles a 10.40 y 10.00 en los machos y hembras infectados, respectivamente). Del mismo modo, los bioensayos revelaron una reducción significativa en la descendencia que apunta a una reducción de la progenie inducida horizontalmente. Estos resultados validan el potencial de la cepa de $B$. pseudobassiana, que podría ser utilizado mediante técnicas de autodiseminación en el control integrado de los vectores del nematodo de la madera del pino.

\section{Bibliografía}

Daub, M., 2008. Investigations on pathogenicity, invasion biology and population dynamics of the pine wood nematode Bursaphelenchus xylophilus (Steiner und Buhrer 1934) 
Nickle 1970 in European conifers. Julius Kühn-Institut, Braunschweig.

Draganova S, Takov D, Pilarska D, Doychev D, Mirchev P, Georgiev G, 2013. Fungal Pathogens on Some Lepidopteran Forest Pests in Bulgaria. Acta Zool. Bulg. 65, 179186.

Dyer LJ, Seabrook WD, 1975. Sensillae on antennal flagellum of sawyer beetles Monochamus notatus (Drury) and Monochamus scutellatus (Say) (Coleoptera-Cerambycidae). J. Morphol. 146, 513-531. DOI: 10.1002/jmor.1051460407.

Espárrago, G., 2012. Resultados de trampeos en Sierra de Dios Padre 2011. Informe I e Informe II. Junta de Extremadura. Presented at the VII Taller de Feromonas de Escolítidos.

Evans HF, McNamara DG, Braasch H, Chadoeuf J, Magnusson C, 1996. Pest risk analysis (PRA) for the territories of the European Union (as PRA area) on Bursaphelenchus xylophilus and its vectors in the genus Monochamus. EPPO Bulletin 26, 199-249.

Graham EE, Poland TM, McCullough DG, Millar JG, 2012a. A comparison of trap type and height for capturing cerambycid beetles (Coleoptera). J. Econ. Entomol. 105, 837-846.

Graham EE, Poland TM, 2012b. Efficacy of fluon conditioning for capturing cerambycid beetles in different trap designs and persistence on panel traps over time. J. Econ. Entomol. 105, 395-401.

Ibeas F, Gallego D, Diez JJ, Pajares JA, 2007. An operative kairomonal lure for managing pine sawyer beetle Monochamus galloprovincialis (Coleoptera: Cerymbycidae). J. Appl. Entomol. 131, 13-20. DOI: 10.1111/j.1439-0418.2006.01087.x.

Ibeas F, Diez JJ, Pajares JA, 2008. Olfactory sex attraction and mating behaviour in the pine sawyer Monochamus galloprovincialis (Coleoptera: Cerambycidae). J. Insect Behav. 21, 101-110. DOI: 10.1007/s10905-007-9104-1.

Lindgren BS, 1983. A multiple funnel trap for scolytid beetles (Coleoptera). Can. Entomol. 115, 299-302.

Naves PM, Camacho S, De Sousa EM, Quartau JA, 2007. Transmission of the pine wood nematode Bursaphelenchus xylophilus through feeding activity of Monochamus galloprovincialis (Col., Cerambycidae). J. Appl. Entomol. 131, 21-25. doi:10.1111/ j.1439-0418.2006.01111.x

Pajares JA, Ibeas F, Diez JJ, Gallego D, 2004. Attractive responses by Monochamus galloprovincialis (Col., Cerambycidae) to host and bark beetle semiochemicals. J. Appl. Entomol. 128, 633-638. DOI: 10.1111/j.1439-0418.2004.00899.633-638.

Pajares JA, Álvarez G, Ibeas, F, Gallego, D, Hall, DR, Farman, DI, 2010. Identification and field activity of a male-produced aggregation pheromone in the pine sawyer beetle, Monochamus galloprovincialis. J. Chem. Ecol. 36, 570-583.

Pajares JA, Álvarez G, Hall DR, Douglas P, Centeno F, Ibarra N, Schroeder M, Teale SA, Wang Z, Yan S, Millar JG, Hanks LM, 2013. 2-(Undecyloxy)-ethanol is a major component of the male-produced aggregation pheromone of Monochamus sutor. Entomol. Exp. Appl. 149, 118-127.

Peng F, Gardescu S, Hajek AE, 2011. Transmission of Metarhizium brunneum conidia between male and female Anoplophora glabripennis adults. BioControl. 56, 771-780.

Rassati D, Toffolo EP, Battisti A, Faccoli M, 2012. Monitoring of the pine sawyer beetle Monochamus galloprovincialis by pheromone traps in Italy. Phytoparasitica 40, 329-336.

Rodrigues, J.M., 2008. National eradication programme for the pinewood nematode, in: 
Pine Wilt Disease: A worldwide threat to forest ecosystems, Ed by Mota, M., Vieira, P., Springer Netherlands, pp. 5-14.

Schroeder M, 2012. Strategies for detection and delimitation surveys of the pine wood nematode in Sweden. Swedish Board of Agriculture, Report 2012 4, 36.

Sevim A, Demir I, Demirbag Z, 2010. Molecular characterization and virulence of Beauveria spp. from the pine processionary moth, Thaumetopoea pityocampa (Lepidoptera: Thaumetopoeidae). Mycopathologia. 170, 269-277.

Shimazu M, 2004. Effects of temperature on growth of Beauveria bassiana F-263, a strain highly virulent to the Japanese pine sawyer, Monochamus alternatus, especially tolerance to high temperatures. Appl. Entomol. Zool. 39, 469-475. doi:10.1303/ aez.2004.469.

Shin, S., 2008. Pine wilt disease in Korea, in: Pine wilt disease, Ed. by Zhao, B.G., Futai, K., Sutherland, J.R., Takeuchi, Y., Springer, Japan, 26-32.

Sousa E, Bravo MA, Pires J, Naves P, Penas AC, Bonifacio L, Mota MM, 2001. Bursaphelenchus xylophilus (Nematoda: Aphelenchoididae) associated with Monochamus galloprovincialis (Coleoptera: Cerambycidae) in Portugal. Nematology 3, 89-91.

Wingfield, M.J., 1982. Association of pine wood nematode with stressed trees in Minnesota, Iowa and Wisconsin. Plant Dis. 934-937.

Zhao, B., 2008. Pine Wilt Disease in China, in: Pine wilt disease, Ed. by Zhao, B.G., Futai, K., Sutherland, J.R., Takeuchi, Y., Springer, Japan, 18-25. 


\section{Datos de la tesis}

Premio Universitario de la SECF 2015 a la mejor tesis doctoral.

Director: Juan A. Pajares.

Centro: Instituto de Investigación en Gestión Forestal Sostenible.

Universidad de Valladolid

Fecha de defensa: Septiembre de 2015

\section{Publicaciones derivadas de la tesis doctoral}

G. Álvarez-Baz, M. Fernández-Bravo, J. Pajares \& E. Quesada-Moraga, 2015. Potential of native Beauveria pseudobassiana strain for biological control of Pine Wood Nematode vector Monochamus galloprovincialis. Journal of Invertebrate Pathology 132 (2015), 48-56 doi:10.1016/j.jip. 2015.08.006

G. Álvarez, D. Gallego, D.R. Hall, H. Jactel, J.A. Pajares, 2015. Combining pheromone and kairomones for effective trapping of the Pine Sawyer Beetle Monochamus galloprovincialis. Journal of Applied Entomology 140 (2015), 58-71. DOI: 10.1111 /jen.12297.

G. Álvarez, B. Ammagarahalli, D.R. Hall, J.A. Pajares, C. Gemeno, 2015. Smoke, pheromone and kairomone olfactory receptor neurons in males and females of the pine sawyer Monochamus galloprovincialis (Olivier) (Coleoptera: Cerambycidae). Journal of Insect Physiology 82 (2015), 46-55. doi:10.1016/ j.jinsphys.2015.08.004

G. Álvarez, I. Etxebeste, D. Gallego, G. David, L. Bonifacio, H. Jactel, E. Sousa \& J.A. Pajares, 2015. Optimization of traps for live trapping of Pine Wood Nematode vector Monochamus galloprovincialis. Journal of Applied Entomology 139 (2015) 618-626

Pajares, J. A., G. Alvarez, D. R. Hall, Ibarra, N., Hoch, G., Halbig, P., Cocos, D., Johansson, H., Schroeder, M., 2016. Attractants for management of the pine sawyer beetle Monochamus sutor, a potential vector of Bursaphelenchus xylophilus. Journal of Applied Entomology. doi: 10.1111/jen.12320 